\title{
Horarios de alimentación y sueño en adolescentes chilenos de San Antonio, V Región: su asociación con obesidad y distribución de adiposidad corporal
}

\author{
Feeding hours and sleep in chilean \\ adolescents of San Antonio, \\ V Region: it association with obesity \\ and corporal adiposity distribution
}

\begin{abstract}
Background: Obesity is a global health problem. Eating breakfast maybe associated with a lower risk of obesity in adolescents; scarce evidence is available in our country about risk of obesity associated to the frequency of daily meals or shortened sleep. Objective: To evaluate the association among daily meals and sleeping schedules and risk of adiposity and obesity in Chilean adolescents. Subjects and methods: In a cross-sectional study, 290 adolescents (12- 19 y), attending to a public school in San Antonio city Chile, were evaluated for nutritional status, daily meal schedule and sleep. Results: Obesity was greater in females consuming $\leq 3$ meals/day vs $\geq 4$ meals/day $(9.4 \%$ vs $0.7 \%$; OR= 7.6 IC95\%:1.8-44.0); also in males $(17.7 \%$ vs $2.6 \%$; OR=9.8 IC 95\%:2.9-41.3). Abdominal circumference was higher in females consuming $\leq 3$ meals/day than those with $\geq 4$ meals/day $(83.8 \pm 10.9$ vs $73.3 \pm 6.6 \mathrm{~cm} ;(p<0.05)$, and also in males $(81.2 \pm$ 9.5 vs $75.5 \pm 6.3 \mathrm{~cm} ; p<0.05) ; 38.8 \%$ of males with $\leq 3$ meals/day had increased body fat vs $7.2 \%$ in those consuming $\geq 4$ meals/day $(O R=10.5$ IC95\%: 3.3-35.1). Males sleeping < $9 \mathrm{~h} / \mathrm{d}$ exhibited $>$ BMI: $22.8 \pm 3.9$ vs $21.5 \pm 3.9 \mathrm{Kg} / \mathrm{m}^{2}(p<0.05)$. Multivariate analysis showed the number of daily meals as the only factor associated with obesity. Conclusions: In these Chilean adolescents, low amount of daily meals is the main factor associated to obesity, to adiposity and to abdominal circumference, and few hours of sleep/day only for males.

Key words: obesity, adiposity, meals, sleep, adolescents.
\end{abstract}

\section{INTRODUCCIÓN}

La obesidad infantil es uno de los mayores problemas de salud pública en Chile. El mapa nutricional de JUNAEB del año 2011, mostraba a nivel nacional que en primer año básico un $22,1 \%$ de niños tenía obesidad (IMC/E > 2 DS). En la comuna de San Antonio, de Chile, la cifra de obesidad en los niños de primer año básico el año 2011 fue 25,5\%; en adolescentes de primer año medio, se apreciaba que 8,9\% tenía obesidad (8,2\% a nivel nacional).

En estas prevalencias en adolescentes hay factores
Karen Pontigo Lues (1) Carlos Castillo-Durán (2)

(1) Instituto de Nutrición y Tecnología de los Alimentos (INTA), Universidad de Chile, Santiago, Chile (2) Departamento de Pediatría, Facultad de Medicina Campus Centro, Universidad de Chile, Santiago, Chile

Dirigir la correspondencia a: Carlos Castillo Durán Departamento de Pediatría Facultad de Medicina Campus Centro Universidad de Chile Santa Rosa 1234, Santiago, Chile Correo electrónico: ccastd@med.uchile.cl

Este trabajo fue recibido el 26 de Junio de 2015 , aceptado con modificaciones el 12 de Enero de 2016 y aceptado para ser publicado el 10 de Marzo de 2016. ambientales asociados, como la disminución de la actividad física, el aumento del tiempo ocupado frente a una pantalla (televisión, computador y videojuegos), el aumento de consumo y disponibilidad de alimentos densos en energía, entre otros $(1,2)$.

Estudios descriptivos estadounidenses han mostrado una disminución en el consumo de desayuno entre los años 1965 y 1991 y una aparente asociación con el aumento de obesidad, particularmente en adolescentes de 15 a 18 años $(3,4)$. En Chile, según la JUNAEB, $45 \%$ de los escolares no tomaba 
desayuno diariamente. La Encuesta Nacional de Consumo Alimentario (ENCA, 2010-2011) (5), reveló un cambio de distribución de comidas de los chilenos, $82 \%$ de los chilenos "tomaba once" (horario de media tarde) a las 20:00 horas, en cambio, la cena estaba presente sólo en $29 \%$ de los chilenos y el almuerzo se observaba en $96 \%$. El desayuno en tanto tuvo una leve disminución, de 94\% que registró la Encuesta Nacional de Vida y Salud del Ministerio de Salud en 2000, a 90\% en este estudio.

La adolescencia es un periodo de transición fisiológica, cognitiva, social y cultural, con cambios frecuentes en los patrones de alimentación: colaciones poco saludables, saltarse comidas, comer fuera del hogar, consumir comida rápida y realizar dietas, especialmente entre las mujeres.

Estudios prospectivos estadounidenses han documentado una asociación inversa entre frecuencia de ingesta de desayuno e índice de masa corporal, incluso ajustando por variables como sexo, edad, nivel socioeconómico y variables psicosociales (6-8).

Por otra parte, se ha observado una asociación inversa entre frecuencia de comidas diarias y la prevalencia de obesidad en adolescentes $(9,10)$. No hay suficiente evidencia en nuestro medio acerca del número de alimentaciones y su asociación con el estado nutricional.

Otro factor que puede influir en la ingesta dietaria de los adolescentes es el número de horas de sueño. La tendencia a una menor duración del sueño en los adolescentes se ha desarrollado en el mismo periodo que el dramático aumento de la prevalencia de obesidad (10). Estudios epidemiológicos en distintas poblaciones han demostrado que un menor número horas de sueño se asocia negativamente con el IMC y determina un mayor riesgo de obesidad (11,12). También hay evidencia que sugiere una consistente relación entre una duración corta del sueño y la adiposidad corporal central (12-14).

El objetivo de este estudio fue analizar la asociación entre horarios de alimentación y de sueño, con el riesgo de obesidad y de adiposidad central o periférica aumentadas, en adolecentes chilenos de la ciudad de San Antonio.

\section{SUJETOS Y MÉTODOS}

Se realizó un estudio transversal en adolescentes pertenecientes a un estrato socioeconómico bajo, asistentes a un colegio municipal de San Antonio, V Región de Chile, entre los años 2011 y 2012. Se incluyeron adolescentes de ambos sexos, con edades entre 12 y 19 años, después de firmar con sus padres un consentimiento y asentimiento informados según pautas del Comité de Ética del INTA. Los criterios de exclusión fueron: enfermedad crónica, trastorno metabólico asociado a obesidad, trastorno de conducta alimentaria, tratamiento con medicamentos o participación de algún tipo de intervención nutricional por alguna patología ya diagnosticada.

El tamaño muestral se calculó en base al estudio de Sjoberg et al (15). Para la variable frecuencia de consumo de desayuno, se consideró la prevalencia mínima de 65\%. Se consideró un poder de $80 \%$ y un error alfa de $5 \%$. La muestra requerida para realizar este estudio fue de 245 adolescentes y se consideró un $15 \%$ de posibles pérdidas.

Las variables de resultado principales fueron el índice de masa corporal, la circunferencia abdominal y los pliegues cutáneos. La estatura se midió en balanza de brazo (SECA), el peso en la misma balanza; la circunferencia abdominal se midió sobre las crestas ilíacas y horizontal al ombligo, con cinta métrica plástica (SECA). La variable índice de masa corporal se analizó de forma continua y categórica como estado nutricional, de acuerdo al IMC del referente CDC-NCHS, en obesidad (IMC > o igual al p95), riesgo de obesidad (IMC entre p85 y p95), normal (IMC entre p10 y p85), según sexo y estadios de Tanner. El porcentaje de grasa corporal se calculó a través de la ecuación de Deurenberg y Westrate, que utiliza la sumatoria de 4 pliegues cutáneos (bicipital, tricipital, suprailíaco, subescapular) y se analizó en forma categorizada como grasa corporal total aumentada (> percentil 90) o normal. Se consideró aumentada en los sujetos con $\geq 30 \%$, por ser el punto de corte asociado al mayor riesgo biológico en población infantil (15). La circunferencia abdominal, se analizó en forma categórica como obesidad abdominal o no, definida como la circunferencia abdominal $\geq$ p90 del referente NHANES III, por sexo y edad (16).

Para la frecuencia de desayuno, se dividieron en los que tomaban desayuno en forma regular ( $\geq 5$ veces/semana) e irregular (< 5 veces/semana). Para el número de comidas diarias principales se clasificaron en 2 grupos: $\leq 3$ comidas/ día y = 4 comidas/día (desayuno, almuerzo, horario de tarde (once) y cena. Según el número de horas de sueño nocturno se clasificaron en 2 grupos: duración corta de sueño ( $<9$ h/ noche) y normal ( $\geq 9 \mathrm{~h} /$ noche) (17).

Se efectuó una encuesta por un entrevistador en un día de la semana, consultando sobre la frecuencia con que el adolescente tomaba desayuno, la calidad del desayuno (lácteos, cereales, fruta, golosinas, infusiones de té o café), el número de comidas diarias principales (no se consideró comidas y golosinas a deshoras), el tipo de familia (mono o biparental), el número de hermanos, la actividad física extraescolar (tipo y frecuencia), la escolaridad parental (mayor grado de uno de los padres), el consumo de tabaco, factores psicosociales como sensación de bienestar con el peso corporal, realizar dietas para controlar el peso, saltarse comidas; factores familiares como el comer en familia, tomar desayuno con alguno de los padres y hábitos de sueño (horas de sueño diurno y nocturno, en días de la semana y fines de semana).

Luego, se realizó un examen físico médico con medición del peso, talla, circunferencia abdominal y pliegues cutáneos.

El foco central del análisis fue examinar la asociación entre la frecuencia del consumo de desayuno y la frecuencia de comidas diarias y medidas de adiposidad como IMC, circunferencia abdominal y adiposidad corporal total, así como la asociación de éstas con el número de horas de sueño nocturno. Se utilizó chi-cuadrado para analizar estadísticamente las asociaciones entre las variables categóricas. Para la comparación de 2 grupos se aplicó la prueba t de Student (índice de masa corporal, porcentaje de grasa corporal y perímetro abdominal entre los que tomaban o no desayuno regular) y para comparar más de 2 grupos se aplicó ANOVA. Se utilizaron modelos de regresión lineal para evaluar la importancia de las variables independientes en el índice de masa corporal, circunferencia abdominal y grasa corporal total. La escolaridad parental, actividad física, tabaco, alcohol, tipo de familia y factores psicosociales fueron evaluadas como potenciales variables confundentes de la relación entre la frecuencia de consumo de desayuno y el número de comidas diarias e índice de masa corporal y adiposidad corporal.

El estudio fue previamente aceptado por el Comité de Ética en Investigaciones del INTA de la Universidad de Chile y se solicitó consentimiento y asentimiento escrito a los padres y niños. Los datos fueron procesados en una planilla EXCEL y en STATA 11.0. 


\section{RESULTADOS}

Los sujetos encuestados fueron 303, de los cuales 298 tenían toda la información requerida; 8 adolescentes no aceptaron participar del estudio, por lo que la muestra final estuvo constituida por 290 adolescentes; de estos 138 (47,6\%) eran mujeres y 152 (52,4\%) varones, con una mediana de 14 años (límites: 12-19 años).

El $83 \%$ de las mujeres era sedentaria y $49 \%$ de los varones (datos no analizados en este estudio). En relación al tabaquismo, no fumaba $80 \%$ de las mujeres y $85 \%$ de los varones, pero en el grupo de 10 a 14 años las mujeres fumaban significativamente más que los varones (tabla 1).

Del total, 58,3\% tenían un estado nutricional normal, $1,7 \%$ eran bajo peso, $25,9 \%$ estaba con sobrepeso y $14,1 \%$ eran obesos. El 11,7\% de los individuos tenía una circunferencia abdominal $\geq$ p90, de acuerdo al referente de Fernández et al (14), mujeres: 10,1\%, varones: 13,2\% (NS).

La grasa corporal total en mujeres fue $35,7 \pm 5,7 \%$ y en varones $29,2 \pm 6,5 \%(p<0,05)$ (tabla 2$)$. Al agrupar por edades, se mantuvo esta diferencia. En toda la muestra, $64,8 \%$ tenía aumento de la grasa corporal total.

Casi $60 \%$ de los adolescentes tenía un patrón de alimentación $\leq 3$ comidas/día (mujeres: 59,4\%, varones: 59,2\%, NS), con diferencia entre grupos etarios (27,9\% en grupo de 10 a 14 años vs 31,3\% en el de 15 a 19 años, p<0,05). El 55\% del total no tomaba desayuno en forma regular (mujeres: $58 \%$, varones: $52 \%)$

En relación a la duración del sueño nocturno, 70\% dormía $<9$ h/día, sin diferencias por sexo. En el grupo de entre 10 y 14 años, 30\% dormía < 9 h/día, comparado con $42 \%$ de los adolescentes entre 15 y 19 años $(p<0,05)$.

En los adolescentes que tomaban desayuno regularmente, la prevalencia de obesidad fue $5,2 \%$ y en los que lo hacían de manera irregular 8,9\% (NS). Sin embargo, el IMC en mujeres que tomaban desayuno en forma regular fue 22,6 $\pm 3,1 \mathrm{Kg} / \mathrm{m}^{2}$ y en las que tomaban desayuno de forma irregular,

\section{TABLA 1}

Características generales de la muestra, según sexo.

\begin{tabular}{|c|c|c|}
\hline & $\begin{array}{c}\text { Mujeres n (\%) } \\
n=138\end{array}$ & $\begin{array}{c}\text { Varones } n(\%) \\
n=152\end{array}$ \\
\hline \multicolumn{3}{|l|}{ Familia } \\
\hline Monoparental & $53(38)$ & $44(29)$ \\
\hline Biparental & $85(62)$ & $108(71)$ \\
\hline Sedentarismo & $115(83)^{*}$ & $74(49)^{*}$ \\
\hline Tabaco & $27(20)$ & $22(14)$ \\
\hline \multicolumn{3}{|l|}{ Educación parental } \\
\hline Básica & $33(24)$ & $31(20)$ \\
\hline Media & $105(76)$ & $121(80)$ \\
\hline Sensación de bienestar de peso & $25(18) *$ & $66(43) *$ \\
\hline Dietas el año anterior & $41(30)^{*}$ & $24(16)^{*}$ \\
\hline
\end{tabular}

*Diferencias significativas entre varones y mujeres chi cuadrado, $p<0,05$.

TABLA 2

Mediciones antropométricas y horas de sueño nocturno, según sexo y edad.

\begin{tabular}{|c|c|c|c|c|}
\hline & & Mujeres $(\mathrm{N}=138)$ & Varones $(\mathrm{N}=152)$ & \\
\hline & Edad & Media (DS) & Media (DS) & $P<*$ \\
\hline \multirow[t]{2}{*}{ Talla/edad pje z } & $10-14$ & $0,1 \pm 1,2$ & $-0,2 \pm 1,1$ & 0,01 \\
\hline & $15-19$ & $0,0 \pm 1,0$ & $-0,3 \pm 1,0$ & 0,06 \\
\hline \multirow[t]{2}{*}{ IMC/edad pje z } & $10-14$ & $1,1 \pm 1,1$ & $0,6 \pm 0,9$ & 0,01 \\
\hline & $15-19$ & $0,9 \pm 1,1$ & $0,7 \pm 0,9$ & 0,1 \\
\hline \multirow[t]{2}{*}{$\mathrm{CA}^{* *}(\mathrm{~cm})$} & $10-14$ & $77,9 \pm 9,2$ & $78,3 \pm 11,5$ & 0,74 \\
\hline & $15-19$ & $79,6 \pm 8,4$ & $80,8 \pm 9,7$ & 0,64 \\
\hline $\mathrm{GCT} * *$ & $10-14$ & $33,7 \pm 5,8$ & $28,9 \pm 7,1$ & 0,0001 \\
\hline (\% > perc. 90$)$ & $15-19$ & $37,2 \pm 5,1$ & $29,5 \pm 5,9$ & 0,0000 \\
\hline Sueño nocturno & $10-14$ & $8,2 \pm 1,1$ & $8,4 \pm 0,9$ & 0,345 \\
\hline (hs) & $15-19$ & $7,8 \pm 1,1$ & $7,7 \pm 1,1$ & 0,389 \\
\hline
\end{tabular}

*Diferencia entre grupos t Student, $\mathrm{p}<$

**IMC: índice de masa corporal; CA: circunferencia abdominal; GCT: grasa corporal total por medición de 4 pliegues cutáneos. 
fue $23,9 \pm 3,7 \mathrm{Kg} / \mathrm{m}^{2}(\mathrm{p}=0,06)$. En varones no hubo diferencias $\left(22,3 \pm 4,1\right.$ vs $\left.22,6 \pm 3,9 \mathrm{Kg} / \mathrm{m}^{2} ; \mathrm{NS}\right)$.

La prevalencia de obesidad abdominal o de adiposidad general aumentada según regularidad o irregularidad del desayuno no fue significativamente diferente. En la frecuencia de comidas diarias, se apreció que en mujeres que consumían $\leq 3$ comidas/ día, 9,4\% era obesa vs $0,7 \%$ en las que consumían $\geq 4$ comidas/día (OR=7,6; IC95\%: 1,8-44,0) (figura 1a). En varones que consumían $\leq 3$ comidas/día, $17,7 \%$ era obeso vs $2,6 \%$ en los que consumían $\geq 4$ comidas (OR=9,8; IC95\%: 2,9-41,3) (figura 1b). El IMC en mujeres que comían $\leq 3$ comidas/día, fue $24,5 \pm 3,7 \mathrm{Kg} / \mathrm{m}^{2}$ vs $21,7 \pm 2,3 \mathrm{Kg} / \mathrm{m}^{2}$ el de las que comían $\geq 4$

\section{FIGURA 1A}

Asociación entre número de comidas diarias y estado nutricional en mujeres adolescentes, según subgrupos de eda.

30

25

20

15

10

5

0

$\leq 310-\quad \leq 315-\quad \geq 410-\quad \geq 415-$

14 años 19 años 14 años 19 años

${ }^{*} \mathrm{p}<0,05$

Normal a obesos

\section{FIGURA 1B}

Asociación entre número de comidas diarias y estado nutricional en varones adolescentes, según subgrupos de edad.

$\%$

30

25

20

15

10

0

${ }^{*} p<0,001$

\section{7}

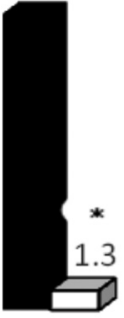

(9) 
$(p<0,05)$. Lo mismo ocurrió en varones $(24,2 \pm 3,9$ vs 19,8 $\left.\pm 2,3 \mathrm{Kg} / \mathrm{m}^{2} ; \mathrm{p}<0,05\right)$.

La obesidad abdominal en mujeres que consumían $\leq 3$ comidas/día fue mayor que la de las que comían $\geq 4$ veces/ día (10,1 vs 3,6\%; $p<0,05)$ (figura 2a), lo mismo en varones $(13,2$ vs $3,3 \% ; p<0,05)$ (figura $2 b)$. En mujeres que consumían $\leq 3$ comidas/día, 52,9\% tenía adiposidad corporal aumentada vs $32,6 \%$ en las que consumían $\geq 4$ comidas/día; estadísticamente significativo sólo en el grupo de 10 a 14 años $(25,3$ vs $8 \%$; OR= 4,2 IC95\% (1,0-18,1). También fue significativa la diferencia en varones $(38,8$ vs $7,2 \%$; OR $=10,5 ;$ IC95\%: $3,3-35,1)$. En términos de $\%$ del peso corporal, la adiposidad general en mujeres fue $36,9 \pm 5,5 \%$ vs $33,9 \pm 5,5 \%$; $p<0,05$ ) y en varones de $31,9 \pm 5,9$ vs $25,2 \pm 5,4 \%$; $(p<0,05)$.

Las mujeres que dormían < de 9 horas diarias, tenían 7,3\% de obesidad vs $2,9 \%$, en las que dormían $\geq 9$ horas (NS). No fue significativa la diferencia en varones $(14,5 \% \mathrm{v} / \mathrm{s} 3,3 \%)$. El IMC en mujeres que dormían $<9$ horas fue $23,4 \pm 3,3 \mathrm{Kg} / \mathrm{m}^{2}$ vs $23,2 \pm 4,1 \mathrm{Kg} / \mathrm{m}^{2}$ en las que dormían $\geq 9$ horas (NS). En cambio, en hombres que dormían < 9 horas el IMC fue 22,8 $\pm 3,9 \mathrm{Kg} / \mathrm{m}^{2}$, y en los que dormían $\geq 9$ horas $21,5 \pm 3,9 \mathrm{Kg}$ / $\mathrm{m}^{2}(\mathrm{p}<0,05)$.

La obesidad abdominal fue semejante en mujeres que dormían $<9$ vs las que dormían $\geq 9$ horas $(6,5 \%$ vs 3,6\%, NS), lo mismo en varones $(9,9 \%$ vs $3,3 \%$, NS). La circunferencia abdominal en varones que dormían $<9$ horas diarias era de $80,4 \pm 10,5 \mathrm{~cm}$, en cambio, en los que dormían $\geq 9$ horas era de $77,5 \pm 11,0 \mathrm{~cm},(p=0,07)$. En mujeres, la circunferencia abdominal en las que dormían $<9$ horas era de 78,6 $\pm 8,4$ $\mathrm{cm}$, y en las que dormían $\geq 9$ horas, de 79,5 $\pm 9,7 \mathrm{~cm}$ (NS).

Al realizar diversos ajustes al modelo de regresión múltiple, sólo apareció como significativa la variable de frecuencia de comidas diarias para explicar un índice de masa corporal o circunferencia abdominal aumentados, controlando por otras variables: sexo, frecuencia de consumo de desayuno, sedentarismo, tabaquismo, comidas familiares, horas de sueño, educación parental. Lo mismo ocurrió al realizar un ajuste del modelo de regresión para explicar la variabilidad de la adiposidad corporal, apareció como significativa la misma variable (frecuencia de comidas diarias) y se agregó sexo y número de horas de sueño (tabla 3).

\section{DISCUSIÓN}

La frecuencia de saltarse el desayuno en forma regular en estos adolescentes fue mayor que la conocida a nivel nacional el año 2011 (74\% vs 43\%) y la de otros datos existentes en Chile (15-19\% omitía el desayuno); también a diferencia de otros estudios nacionales saltarse el horario de cena fue menor. Debe tomarse en cuenta que es difícil comparar frecuencias con otros estudios, debido a las distintas metodologías y definiciones utilizadas.

La prevalencia de obesidad en esta muestra fue 14\%. La prevalencia de obesidad no fue significativamente diferente entre el grupo que consumía desayuno regularmente y en el grupo que no lo hacía, a diferencia de lo demostrado en algunos estudios $(6-8,18-20)$. En este estudio, la prevalencia de saltarse el desayuno fue mayor en adolescentes mujeres, concordante con estudios previos $(4,8,18)$. Hubo una tendencia a ser significativa la asociación de frecuencia de consumo de desayuno irregular con IMC elevado en mujeres, lo cual se ha demostrado en varios estudios, no así en nuestro medio (18-20).

En relación a la frecuencia de comidas diarias, ésta se asoció de manera significativa con obesidad en hombres y mujeres, en ambos grupos etáreos. Esto concuerda con estudios previos que demuestran esta asociación inversa en adolescentes $(8,9)$.

En este estudio, el riesgo de obesidad en varones fue 9 veces mayor en los adolescentes que consumían $\leq 3$ comidas diarias y en mujeres, fue de 7 veces más. El IMC fue significativamente menor en los que consumían $\geq 4$ comidas diarias. Esto concuerda con las escasas publicaciones al respecto en este grupo etario $(8,9)$. El hecho de tener menor número de comidas diarias implica muchas veces, tener comidas a deshoras, que incluyen alimentos poco saludables (golosinas, bebidas). Se destaca además que la prevalencia de obesidad abdominal fue mayor en los adolescentes que consumían $\leq 3$ comidas al día. Al comparar los porcentajes de grasa corporal total, también se apreció que fueron mayores cuando los adolescentes consumían $\leq 3$ comidas diarias, y la prevalencia de grasa corporal aumentada fue mayor en todos los grupos, excepto en el grupo mujeres de 15 a 19 años. Esto podría deberse a la composición corporal propia de la edad (aumento de la masa grasa en mujeres). Dada la falta de evidencia en esta área, es

\section{TABLA 3}

Análisis de regresión múltiple para valorar la influencia de cada variable sobre estado nutricional, circunferencia abdominal y adiposidad general corporal.

\begin{tabular}{|c|c|c|c|c|c|c|c|c|c|}
\hline & \multicolumn{3}{|c|}{$\begin{array}{l}\mathrm{IMC} \\
\mathrm{r}^{2}=0,28\end{array}$} & \multicolumn{3}{|c|}{$\begin{array}{l}\text { Obesidad abdominal } \\
r^{2}=0,11\end{array}$} & \multicolumn{3}{|c|}{$\begin{array}{l}\text { Adiposidad corporal } \\
r^{2}=0,27\end{array}$} \\
\hline & $B^{*}$ & $\mathrm{DE}$ & $\mathrm{p}$ & B & $\mathrm{DE}$ & $p$ & $B$ & $\mathrm{DE}$ & $\mathrm{P}^{* *}$ \\
\hline _cons & 1,91 & 0,11 & 0,00 & 0,20 & 0,05 & 0,00 & 1,05 & 0,07 & 0,00 \\
\hline Sexo & 0,02 & 0,07 & 0,07 & 0,03 & 0,04 & 0,33 & $-0,39$ & 0,04 & 0,00 \\
\hline Desayuno & $-0,01$ & 0,07 & 0,95 & 0,04 & 0,04 & 0,22 & $-0,02$ & 0,04 & 0,66 \\
\hline Comidas diarias & $-0,81$ & 0,07 & 0,00 & $-0,19$ & 0,04 & 0,00 & $-0,29$ & 0,04 & 0,00 \\
\hline Horas de sueño & $-0,04$ & 0,08 & 0,57 & 0,01 & 0,03 & 0,96 & $-0,12$ & 0,05 & 0,02 \\
\hline Escolaridad padres & $-0,07$ & 0,09 & 0,42 & $-0,06$ & 0,04 & 0,12 & $-0,04$ & 0,05 & 0,46 \\
\hline
\end{tabular}


difícil comparar estos resultados con otras investigaciones.

Otro factor que puede influir en la ingesta dietaria de los adolescentes es el número de horas de sueño. En nuestro estudio se apreció que hubo una asociación inversa entre horas de sueño e IMC en varones, no así en mujeres, lo que concuerda con algunos estudios publicados que demuestran la asociación entre duración corta de sueño e IMC significativa sólo en varones adolescentes $(12,21)$. Con respecto a la circunferencia abdominal, hubo una tendencia a haber una asociación inversa con horas de sueño sólo en varones. Se ha demostrado que la asociación observada entre duración de sueño e IMC se debe en gran parte a la adiposidad y que esta relación está influenciada por el género masculino en adolescentes $(13,22,23)$. Esta diferencia pudiera ser explicada por los cambios específicos del género en la fisiología y composición corporal de la pubertad.

Es necesario considerar algunas limitaciones en este trabajo. Primero, fue realizado en adolescentes de la $\mathrm{V}$ Región, por lo que no podrían generalizarse estos hallazgos a toda la población chilena, pero sí puede representar los hábitos de comidas de ambientes urbanos que no sea la capital (Santiago); segundo, al ser una encuesta por recordatorio de algunos días, puede no representar los hábitos usuales de la muestra; tercero, el diseño de este estudio fue transversal, por lo que no se puede afirmar causalidad en los hallazgos. Con respecto a la medición de sueño, ésta fue autorreportada, por lo que pudiera ser menos confiable.

En conclusión, los resultados obtenidos demostraron que la mayoría de estos adolescentes chilenos no consumía desayuno regularmente, comía $\leq 3$ comidas/día y dormía $<9$ horas diarias. La menor frecuencia de comidas diarias, más que el no consumir desayuno per se, se asoció con más riesgo de obesidad y mayor adiposidad corporal. El sueño puede representar un factor de riesgo modificable en estrategias para combatir la epidemia de obesidad en adolescentes.

\section{RESUMEN}

Introducción: La obesidad es un problema mundial de salud. El desayuno parece asociarse con un menor IMC en adolescentes, pero hay poca evidencia en nuestro medio del riesgo de obesidad general o abdominal según la cantidad de comidas diarias y las horas de sueño. Objetivo: Estudiar la asociación entre los horarios de alimentación y de sueño y el riesgo de obesidad y adiposidad en adolescentes chilenos. Sujetos y métodos: Estudio transversal en 290 adolescentes (12-19 años) de un colegio municipal de San Antonio, V Región de Chile. Se les evaluó el IMC, la circunferencia abdominal (CA), la adiposidad general (AG) y se completó una encuesta de frecuencia de consumo y sueño. Resultados: En niñas hubo más obesidad en aquellas con $\leq 3$ comidas/día vs con $\geq 4$ comidas/día ( 9,4 vs $0,7 \%$; OR=7,6; IC95\%: $1,8-44,0)$, también en varones (17,7\% vs 2,6\%; OR=9,8; IC 95\%: 2,9$41,3)$. En mujeres la $C A$ fue mayor con $\leq 3$ vs $\geq 4$ comidas/día $(83,8 \pm 10,9$ vs $73,3 \pm 6,6 \mathrm{~cm}$; $p<0,05)$; lo mismo en varones $(81,2 \pm 9,5 \mathrm{~cm}$ vs $75,5 \pm 6,3 \mathrm{~cm} ; \mathrm{p}<0,05)$. Un $38,8 \%$ de los varones tenían AG aumentada con $\leq 3$ comidas/día vs $7,2 \%$ con $\geq 4$ comidas/día (OR=10,5; IC95\%: 3,3-35,1). Los varones que dormían $<9 \mathrm{~h} / \mathrm{d}$ presentaron $>I M C$ que los con $>9 \mathrm{~h} / \mathrm{d}$ : $22,8 \pm 3,9$ vs $21,5 \pm 3,9 \mathrm{Kg} / \mathrm{m}^{2}(\mathrm{p}<0,05)$. El análisis multivariado mostró al numero de comidas/día como el principal factor asociado a obesidad. Conclusiones: En estos adolescentes la frecuencia de comidas $\leq 3 /$ día se asoció con un mayor riesgo de adiposidad y de obesidad central y en varones además con $<9 \mathrm{~h} / \mathrm{d}$ de sueño.
Palabras clave: obesidad, adiposidad, comidas, sueño; adolescentes

Agradecimientos: Agradecemos sinceramente la colaboración de Yasna Orellana y Bárbara Leyton (bioestadísticas del INTA, Universidad de Chile) por su apoyo en los análisis estadísticos y de Luisa Zúñiga, Directora del Liceo Nacional de Llo Lleo, V Región, por su buena disposición y cooperación para acceder a los alumnos.

\section{BIBLIOGRAFÍA}

1. Olivares S, Kain J, Lera L, et al. Nutritional status, food consumption and physical activity among Chilean school children. A descriptive study. Eur J Clin Nutr 2004; 58:1278-85.

2. Burrows $R$, Burgueño $M$, Gattas $V$, Barrera $G$, Leiva L. Biological, familiar and metabolic characteristics of pediatric obesity. Rev Med Chile 2001; 129:1155-62.

3. Wolfe WS, Campbell CC, Frongillo EA, Haas JD, Melnik TA. Overweight schoolchildren in New York State: prevalence and characteristics. Am J Public Health 1994; 84:807-13.

4. Siega- Riz A, Popkin B, Carson T. Trends in breakfast consumption for children in the United States from 1965 to 1991. Am J Clin Nutr. 1998; 67(Suppl.): 748S-56S.

5. Public Health School, Faculty of Medicine, Universidad de Chile. National Food Consumption Survey. 2010-2011. In: www.ispch.cl/noticia/15048.

6. Pereira M, Erickson E, McKee $P$ et al. Breakfast frequency and quality may affect glycemia and appetite in adults and children. J Nutr. 2011; 141: 166S-8S.

7. Timlin $M$, Pereira $M$, Story $M$, Neumark-Sztainer $D$. Breakfast eating and weight change in a 5-year prospective analysis of adolescents: Project EAT (Eating Among Teens). Pediatrics 2008; 121: e638-e45.

8. Antonogeorgos $G$, Panagiotakos DB, Papadimitriou A, Priftis KN, Anthracopoulos M, Nicolaidou P. Breakfast consumption and meal frequency interaction with childhood obesity. Pediatr Obes. 2012; 7:65-72.

9. Mota J, Fidalgo F., Silva $R$, et al. Relationship between physical activity, obesity and meal frequency in adolescents. Ann Hum Biol. 2008; 35: 1-10.

10. Kulovitz MG, Kravitz LR, Mermier $C$, et al. Potential role of meal frequency as a strategy for weight loss and health in overweight or obese adults. Nutrition 2014; 30:386-92.

11. Van Cauter E, Spiegel K, Tasali E, Leproult R. Metabolic consequences of sleep and sleep loss. Sleep Med. 2008; 9 (Suppl 1): S23-S8.

12. Gupta NK, Mueller WH, Chan W, Meininger JC. Is obesity associated with poor sleep quality in adolescents? Am J Hum Biol. 2002; 14:762-8.

13. Knutson $K L$. Sex differences in the association between sleep and body mass index in adolescents. J Pediatr. 2005; 147:830-4.

14. Yunxian Yu, Brandon S. Lu, Binyan Wang, et al. Short sleep duration and adiposity in Chinese adolescents. Sleep 2007; 30:1688-97.

15. Sjoberg A, Hallberg L, Hoglund D, Hulthen L. Meal pattern, food choice, nutrient intake and lifestyle factors in the Goteborg Adolescence Study. Eur J Clin Nutr. 2003; 57:1569-78.

16. Williams D, Going S, Lohman T, et al. Body fatness and risk for elevated blood pressure, total cholesterol and serum lipoprotein ratios in children and adolescents. Am J Public Health 1992; 82: 358-63. 
17. Fernández JR, Reeden DT, Petrobielli A, Allison DB. Waist circumference percentiles in nationally representative sample of African-American, European-American and Mexican American children and adolescents. J Pediatr. 2004; 145:439-44.

18. Iglowstein I, Jenni OG, Molinari L, Largo RH. Sleep duration from infancy to adolescence: reference values and generational trends. Pediatrics 2003; 111: 302-7.

19. Barton BA, Eldridge AL, Thompson D, et al. The relationship of breakfast and cereal consumption to nutrient intake and body mass index: the National Heart, Lung, and Blood Institute Growth and Health Study. J Am Diet Assoc. 2005; 105:1383-9.

20. Affenito SG, Thompson DR, Barton BA, et al. Breakfast consumption by African-American and white adolescent girls correlates positively with calcium and fiber intake and negatively with body mass index. J Am Diet Assoc. 2005; 105:938-945.

21. Berkey CS, Rockett HR, Gillman MW, Field AE, Colditz $G A$. Longitudinal study of skipping breakfast and weight change in adolescents. Int J Obes Relat Metab Disord. 2003; 27:1258-66.

22. Shi Z, Taylor A, Gill T. Short sleep duration and obesity among Australian children. BMC Public Health 2010; 10:609.

23. Rontoyanni VG, Baic S, Cooper AR. Association between nocturnal sleep duration, body fatness and dietary intake in Greek women. Nutrition 2007; 23: 773-7.

24. Stranges S, Cappuccio FP, Kandala NB, et al. Cross-sectional versus prospective associations of sleep duration with changes in relative weight and body fat distribution: The Whitehall II Study. Am J Epidemiol. 2008; 167: 321-9. 